\title{
Morphometric Analysis of the Sub-basin of the Upano Para River Flood Analysis
}

\section{Análisis Morfométrico de la Subcuenca del Río Upano Para Análisis de Inundaciones}

\author{
Diego Shagui Miranda, Mayra Chucay Chacha, and Jose Negrete Costales
}

Escuela Superior Politécnica de Chimborazo Sede Morona Santiago, Facultad de Ciencias de la Carrera de Ingeniería Ambiental, Morona Santiago, Macas, Ecuador

I International Congress of Science and Technology Morona Santiago-CICTMS 2020

Corresponding Author:

Diego Shagui Miranda

diego.shagui@espoch.edu.ec

Published: 29 August 2021

Production and Hosting by

Knowledge E

(c) Diego Shagui Miranda et al. This article is distributed under the terms of the Creative Commons Attribution License, which permits unrestricted use and redistribution provided that the original author and source are credited.

\section{Abstract}

The purpose of this work is to analyze the morphometric characteristics that the Upano River sub-basin presents with the help of SIG tools, which is located in the Ecuadorian Amazon in the Morona Santiago Province. For the analysis of the study, nine morphometric parameters were taken into consideration: area, perimeter, axial length, length of the main channel, total drainage length, average width, shape factor, Gravelius coefficient of compactness or Gravelius index, Drainage density and their results were calculated using ArcGIS ${ }^{\circledR}$ software using the ArcMap $^{\circledR}$ tool. The area of the sub-basin comprises approximately $6075.54 \mathrm{~km}^{2}$ with its main channel $266.73 \mathrm{~km}$ long. The compactness coefficient is related to the concentration time, being a dimensionless value, resulting in 1.35 which presents a round oval to oblong oval shape. From this result, an environmental interpretation was made using the Gravelius index and it was determined that the sub-basin of the Upano River is medium with a tendency to floods.

Keywords: morphometric characteristics; sub-basin, parameters, Gravelius index, drainage density.

\section{Resumen}

La finalidad del presente trabajo es analizar las características morfométricas que presenta la subcuenca del rio Upano con ayuda de herramientas SIG, la cual se encuentra ubicada en la Amazonía ecuatoriana en la Provincia de Morona Santiago. Para el análisis del estudio se tomó en consideración nueve parámetros morfométricas: área, perímetro, longitud axial, longitud del cauce principal, longitud total de drenaje, ancho promedio, factor forma, coeficiente de compacidad de Gravelius o índice de Gravelius, densidad de drenaje y sus resultados fueron calculados por medio del software ArcGIS $^{\circledR}$ mediante la herramienta ArcMap ${ }^{\circledR}$. El área de la subcuenca comprende aproximadamente $6075,54 \mathrm{~km}^{2}$ y su cauce principal de $266,73 \mathrm{~km}$ de longitud. El coeficiente de compacidad está relacionado con el tiempo de concentración siendo un valor adimensional, teniendo como resultado 1,35 lo cual presenta una forma de oval redonda a oval oblonga. A partir de dicho resultado se realizó una interpretación ambiental por medio del índice de Gravelius y se pudo determinar que la subcuenca del Rio Upano es mediana a tendencia a inundaciones.

Palabras Clave: características morfométricas, subcuenca, parámetros, índice de Gravelius, densidad de drenaje. 


\section{Introducción}

Al inicio de la civilización, el ser humano tuvo la incertidumbre de conocer y predecir algunos sucesos propios de la naturaleza con el propósito de aprovechar y manejar de mejor manera los recursos naturales [1] y con el paso del tiempo, el hombre al incorporar la ciencia y la tecnología pudo lograr establecer hasta cierto punto dichos fenómenos [2].

Actualmente el estudio de las cuencas y subcuencas hidrográficas representa una fuente importante de información [3] que proporciona la base para la planificación del manejo de los recursos naturales [4], incluyendo estudios hidrológicos, sociales y ecológicos [5].

El funcionamiento de una subcuenca es similar al de un reservorio debido a que capta la precipitación y una parte de ésta pasa a escurrimiento [6], por lo tanto una subcuenca hidrográfica corresponde al territorio que abarca los ríos secundarios que desembocan en un rio principal de una cuenca [7] todo este proceso de transformación depende de las condiciones climáticas y de las características físicas que presente la subcuenca [8], incluyendo la naturaleza del suelo y la cobertura vegetal [9]. Por lo tanto el análisis morfométrico de una subcuenca es el estudio de variables lineales, de superficie, relieve y drenaje [6], que determina a su vez las características físicas que presenta la subcuenca [10].

Hoy en día con la ayuda de herramientas metodológicas como los Sistemas de Información Geográfica [11], y la interpretación de imágenes satelitales hacen posible que se pueda caracterizar las propiedades morfométricas de las subcuencas hídricas [6].

El objetivo del presente trabajo de investigación es analizar las características morfométricas que presenta la subcuenca del rio Upano para el análisis de inundaciones, el cual se desarrolló con la ayuda de herramientas de los Sistemas de Información Geográfica (SIG).

\section{Materiales y Métodos}

\section{1. Área de estudio}

La subcuenca del rio Upano se encuentra ubicado en la Amazonía ecuatoriana en la Provincia de Morona Santiago.

El río Upano nace del volcán Sangay que atraviesa de Norte a Sur y luego al Este forma el Rio Santiago con la unión del Zamora [12].

La Provincia de Morona Santiago por sus características físicas fluctúa desde los 300 hasta los 2900 metros sobre el nivel del mar, cuenta con una extensión de 24154.55 $\mathrm{km}^{2}$, ubicada en los meridianos $76^{\circ} 05^{\prime}$ y $78-58^{\prime}$ de longitud occidental paralelos 1-25' y $3^{\circ} 54^{\prime}$ al sur de la línea occidental [13]. 


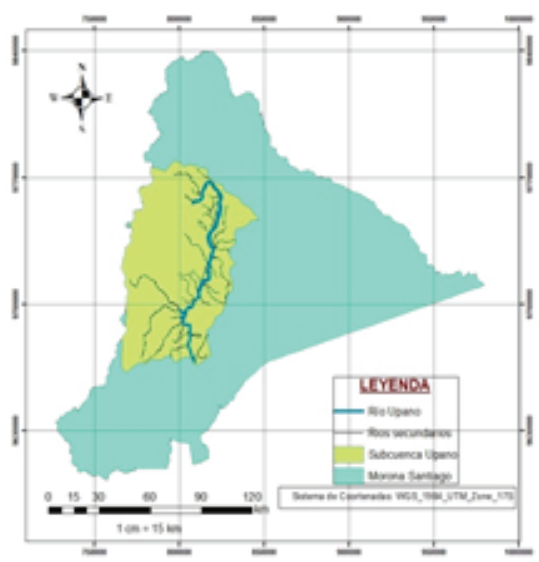

Figure 1

Subcuenca del Río Upano.

Su precipitación es de $2500 \mathrm{~mm}$ media anual, con una temperatura media de $22^{\circ} \mathrm{C}$ teniendo un clima tropical y subtropical, las estaciones son poco marcadas distinguiéndose por su cantidad de precipitaciones pluviométricas. La provincia de Morona Santiago abarca un total de 12 cantones [14].

\subsection{Parámetros morfométricos a considerar en la subcuenca del rio Upano}

\subsubsection{Parámetros de forma}

Área [A] $\left(\mathbf{k m}^{2}\right)$ : el área de una subcuenca se define como la superficie de proyección horizontal, delimitada por la divisoria de agua [15].

Perímetro [P] (km): es la medición de la línea que encierra el área de la subcuenca por la divisoria de aguas [16].

Longitud axial [La] (km): se determina siguiendo el curso de agua más largo correspondiente al drenaje principal desde la desembocadura hasta su nacimiento en línea recta [17].

Longitud del cauce principal (L): se define como la distancia entre la desembocadura y el nacimiento del cauce siguiendo su trayectoria [18].

Longitud total del drenaje [ $\mathbf{L n}](\mathbf{k m})$ : corresponde a la sumatoria de la longitud de todas las corrientes que se unen a la subcuenca [19].

Ancho promedio [Ap] (km): se define como la relación entre el área o superficie (A) y la longitud axial de la cuenca (La).

$$
A p=\frac{A}{L a}
$$

[20]. 
Factor forma (IF): es un factor adimensional que señala como se regula la concentración del escurrimiento superficial en la subcuenca. Se expresa como la relación entre el ancho promedio de la cuenca y la longitud axial [21].

$$
I F=\frac{A p}{L a}=\frac{A}{L a^{2}}
$$

\subsubsection{Forma de la subcuenca en relación a la forma Horton}

Table 1

Relación forma Horton [22, 23].

Rango de valores
$<0.22$
$0.22-0.30$
$0.3-0.37$
$0.37-0.45$
$0.45-0.60$
$0.60-0.80$
$0.80-1.12$
$>1.20$

Forma de la subcuenca
Muy alargada
Alargada
Ligeramente alargada
Ni alargada ni ensanchada
Ligeramente ensanchada
Ensanchada
Muy ensanchada
Completamente redonda

Una cuenca con un IF bajo está menos sujeta a eventos torrenciales que una cuenca con el mismo tamaño pero con un IF mayor. Según [24] el máximo valor de este parámetro corresponde a 0.7854 cuando la subcuenca es completamente redonda, y su valor se va acercando a cero a medida que la subcuenca se torna alargada.

Coeficiente de compacidad de Gravelius (kc): se define como la razón entre el perímetro de la cuenca y el área de la cuenca.

$$
K c=0.28 \frac{P}{\sqrt{A}}
$$

dónde:

$\mathrm{K}_{c}$ : coeficiente de compacidad;

$\mathrm{P}$ : perímetro;

A: área de la cuenca $\left(\mathrm{km}^{2}\right)$.

Los valores obtenidos de este índice nunca serán menores a 1, por lo que mientras más cercano es al 1 mostrara una tendencia a concentrar grandes volúmenes de agua [25].

El Kc está relacionado con el tiempo de concentración, es decir el tiempo que tarda una gota de lluvia en moverse desde la parte más lejana de la subcuenca hasta la desembocadura [26]. 
Table 2

Índice de Gravelius para la evaluación de la forma [27].

Rango de $\mathbf{K}_{C}$
$1-1.25$
$1.25-1.50$
$1.50-1.75$

Forma
Redonda a oval redonda
De oval redonda a oval oblonga
De oval oblonga a rectangular oblonga

Table 3

Índice de Gravelius para la evaluación de una interpretación ambiental [28].

$\mathbf{K}_{c}$
$1-1.25$
$1.25-1.50$
$1.50-1.70$
$>1.70$

\section{Interpretación Ambiental}

Alta tendencia a inundaciones

Mediana tendencia a inundaciones

Baja tendencia a inundaciones

Cuencas propensas a la conservación

\subsubsection{Parámetro de drenaje}

Densidad de drenaje [Dd] $\left(\mathbf{k m}^{-1}\right)$ : se define como la longitud total de los cauces dividida entre el área total de drenaje.

$$
D d=\frac{L n}{A} \quad[6],
$$

dónde:

Ln: longitud total de todos los cauces en $\mathrm{km}$;

A: superficie de la cuenca en $\mathrm{km}^{2}$.

Table 4

Densidad de drenaje [26].

\section{Densidad de drenaje $\left(\mathbf{k m} / \mathbf{k m}^{2}\right)$}

$<1$

1 a 2

2 a 3

$>3$

\section{Categoría \\ Bajo \\ Moderada \\ Alta \\ Muy alta}

Seis de los nueve parámetros establecidos se calcularon mediante el software ArcGIS ${ }^{\circledR}$ por medio de la herramienta ArcMap ${ }^{\circledR}$. Para calcular los parámetros de área y perímetro se necesitó los comandos de: Calculate Geometry, Measure.

Para este trabajo de investigación no se dispuso de datos de entrada, además no se utilizó imágenes satelitales por la extensa área de estudio y por consiguiente las imágenes no se evidenciaban de manera. 


\section{Resultados}

Los resultados morfométricos alcanzados para la subcuenca del río Upano se presentan en la Tabla 5.

Table 5

Resultado de los parámetros morfométricos de la subcuenca del Upano.

Parámetro morfométrico
Área
Perímetro
Longitud axial
Longitud del cauce principal
Longitud total de drenaje
Ancho promedio
Factor forma
Coeficiente de compacidad de Gravelius
Densidad de drenaje

\begin{tabular}{l|l} 
Resultado & Unidades \\
6075.54 & $\mathbf{k m}^{2}$ \\
376.61 & $\mathbf{k m}$ \\
87.43 & $\mathbf{k m}$ \\
266.73 & $\mathbf{k m}$ \\
629.64 & $\mathbf{k m}$ \\
69.49 & $\mathbf{k m}$ \\
0.79 & - \\
1.35 & - \\
0.10 & $\mathbf{k m}^{-1}$
\end{tabular}

Luego de realizar los cálculos correspondientes a los parámetros asociados a la forma de la cuenca Tabla 5 el rio Upano presenta un área de $6075.54 \mathrm{~km}^{2}$, un perímetro de $376.61 \mathrm{~km}$, una longitud axial de $87.43 \mathrm{~km}$, una longitud del cauce principal de 266.73 $\mathrm{km}$ y un ancho promedio de $69.49 \mathrm{~km}$.

El factor forma es un parámetro adimensional por lo que no presenta unidades del cual se obtuvo como resultado un valor de 0.79 , por lo tanto la mencionada subcuenca presenta una forma ensanchada en relación a la Tabla 1 en donde se enfatiza la forma de una subcuenca en relación a Horton.

El coeficiente de compacidad $(\mathrm{Kc})$ está relacionado con el tiempo de concentración siendo un valor adimensional que no presenta unidades teniendo como resultado 1.35 lo cual nos representa que el Rio Upano presenta una forma de oval redonda a oval oblonga.

Según el índice de Gravelius para la evaluación de la forma Tabla 2, a partir del coeficiente de compacidad podemos realizar una evaluación de una interpretación ambiental Tabla 3 , con un valor $\mathrm{K}_{c}$ de 1.35 se determina que el Rio Upano es mediana a tendencia a inundaciones.

En el parámetro asociado a la red de drenaje tenemos el parámetro de densidad de drenaje que es un parámetro físico que refleja la dinámica de la cuenca y el tipo de escorrentía superficial obteniendo como resultado un valor de $0.10 \mathrm{~km}^{-1}$ que nos representa una categoría baja de escorrentía según la densidad de drenaje Tabla 4. 


\section{Discusión}

Por medio los resultados del coeficiente de compacidad de Gravelius según la clasificación propuesta por (Meza M, Rodríguez A, Corvacho O, Tapia A., 2014) es considerada de oval redonda a oval oblonga.

Según (Sellers C, Buján S, Corbelle E, Miranda D., 2017) al tener un bajo valor de densidad de drenaje tiende a acumular agua en la superficie de la subcuenca, por lo tanto concuerda con lo que menciona Verdugo, M. en su investigación 'Análisis morfométrico de las microcuencas a las que pertenece el bosque y vegetación protectora Aguarongo (BVPA), influencia en el comportamiento hidrológico' que al tener un bajo valor de densidad de drenaje va a representar un valor característico para ser considerado como mediana en tendencia a inundaciones.

\section{Conclusión}

La subcuenca del rio Upano al presentar un valor bajo de la densidad de drenaje hace que tenga un alto tiempo de retención de las precipitaciones, lo que favorece a la formación de los humedales.

La subcuenca estudiada presenta un área de $6075.54 \mathrm{~km}^{2}$ y un índice de compacidad de 1.35 lo que se interpreta que es mediana en tendencia a inundaciones.

En la actualidad con el aporte de los Sistemas de Información Geográfica es posible realizar una interpretación de los parámetros morfométricos que intervienen en los estudios de las cuencas y subcuencas, de tal manera que se puede generar respuestas inmediatas.

Este estudio da a conocer aportes considerables e importantes sobre el funcionamiento hidrológico que presenta el rio Upano en la Provincia de Morona Santiago, por lo que se podría tomar en consideración los resultados obtenidos para estudios posteriores, teniendo en cuenta que para obtener mejores resultados es adecuado trabajar con áreas pequeñas.

El trabajo de investigación proporciona información relevante a la localidad del Cantón Morona debido a que el Rio Upano presenta resultados de mediana en tendencia a inundaciones, por lo tanto se podrá evidenciar inundaciones siempre y cuando existan precipitaciones considerables.

\section{References}

[1] xx Vásquez C, Herrera D, Gutierrez Y. Caracterización morfométrica de la cuenca de la quebrada Tatamaco, del municipio Villavieja del departamento del Huila, mediante el uso de la herramienta HEC-GEOHMS. 2014. Available from: https://repository.ucatolica.edu.co/bitstream/10983/ 1719/2/DOCUMENTO\{\%\}2OFINAL\{\%\}20TRABAJO\{\%\}20DE\{\%\}20GRADO.pdf.

[2] Figueroa S, Sánchez G, Vidales A. La ciencia y tecnología en el desarrollo: Una visión desde América Latina. 2009. Available from: http://biblioteca.clacso.edu.ar/Mexico/uacp-uaz/ 20100322012242/CYTED.pdf.

[3] Villota M. Análisis morfométrico de la Cuenca del Río Mulaló utilizando herramientas de SIG. 2019. Available from: https://repository.usta.edu.co/bitstream/handle/11634/16665/2019mariovillota. pdf?sequence=3\&isAllowed=y. 
[4] Rodríguez F. Cuencas hidrográficas, descentralización y desarrollo regional participativo. 2006. Available from: https://www.redalyc.org/pdf/666/66612867008.pdf.

[5] Guevara R, Olguín JL, Mancilla O, Barreto O. Análisis morfométrico de la cuenca hidrográfica del rio Ayuquilla, Jalisco-México. 2019. Available from: https://www.researchgate.net/ publication/338286235_ANALISIS_MORFOMETRICO_DE_LA_CUENCA_HIDROGRAFICA_DEL_ RIO_AYUQUILA_JALISCO-MEXICO.

[6] Gaspari F, Rodriguez A, Senisterra G, Denegri G, Delgado M, Basteiro S. Caracterización morfométrica de la cuenca alta del rio Sauce Grande, Buenos Aires, Argentina. 2012. Available from: http://sedici. unlp.edu.ar/handle/10915/25777.

[7] Avendaño R. Subcuencas y microcuencas hidrográficas. 2016. Available from: $\quad$ https://es.slideshare.net/kimberlynveronica/subcuencas-y-microcuencas? fbclid=IwAR2HMyqVk8zsVELZIx7ICpxe5ILyX07xia8u6hpiMbAThPyC9e-IoKiZXDY\#: \$\{lsim\}\$:text=SUB\{\%\}20CUENCA\{\%\}3A\{\%\}2OSon\{\%\}2Olos\{\%\}2Or\{\%\}C3\{\%\}ADos, alimentan\{\%\}20a\{\%\}2Olos\{\%\}20r\{\%\}C3\{\%\}ADos\{\%\}20secundarios.

[8] Ramsar M. Manejo de cuencas hidrográficas: Integración de la conservación y del uso racional de los humedales en el manejo de las cuencas hidrográficas. 2010. Available from: https://www.ramsar.org/ sites/default/files/documents/pdf/lib/hbk4-09sp.pdf.

[9] Cruz B, Gaspari F, Rodríguez A, Carrillo F, Téllez J. Investigación y Ciencia. 2015. Available from: https://www.redalyc.org/articulo.oa?id=67441039004

[10] Cardona BL. Conceptos básicos de morfometría de cuencas hidrográficas. 2016. Available from: http://www.repositorio.usac.edu.gt/4482/1/Conceptos\%20b\%C3\%A1sicos\%20de\%20Morfometr\%C3\% ADa\%20de\%20Cuencas\%20Hidrogr\%C3\%A1ficas.pdf.

[11] Loro M. Cálculo de cuenca vertiente a partir de un DTM. 2012. Available from: http: //oa.upm.es/19455/1/C\{\%\}C3\{\%\}A1/culo_de_cuenca_vertiente_con_ArcGIS._Por_Manuel_Loro_ \{\%\}282012\{\%\}29._License_Creative_Commons.pdf.

[12] Rostain S, Saulieu GD. El Pastaza y el Upano, dos rios tropicales que conectan los Andes a la Amazonía. 2019. Available from: https://horizon.documentation.ird.fr/exl-doc/pleins_textes/ divers19-08/010076798.pdf.

[13] GADPMS. Información básica de la Provincia de Morona Santiago. 2020. Available from: https: //moronasantiago.gob.ec/informacion-de-morona-santiago/\#.

[14] Noguera J. Plan de Marketing Turístico para el Cantón Morona (Macas), provincia de Morona Santiago [Thesis]. 2009. Available from: http://dspace.uazuay.edu.ec/bitstream/datos/1657/1/07326.pdf.

[15] Aparicio F. Fundamentos de hidrología de superficie. 1992. Available from: https://www.academia.edu/ 8254237/Fundamentos_de_hidrologia_de_superficie_-_Aparicio.

[16] Barrera D, Presutti M. El perímetro de una cuenca hídrica: su tratamiento objetivo en la definición de índices geomorfológicos. 2012. Available from: https://core.ac.uk/download/pdf/84263985.pdf.

[17] POMCA. Caracterización físico-biota de la cuenca. Available from: https://www.car.gov.co/uploads/ files/5c1a9fcf99956.pdf.

[18] Gaspari F, Rodríguez A, Senisterra G, Delgado M, Basteiro S. Elementos metodológicos para el manejo de cuencas hidrográficas. 2013. Available from: http://sedici.unlp.edu.ar/handle/10915/27877.

[19] Ibáñez S, Moreno H, Gisbert J. Morfología de las cuencas hidrográficas. Available from: https://riunet. upv.es/bitstream/handle/10251/10782/Morfolog\{\%\}C3\{\%\}ADa\{\%\}20de\{\%\}20una\{\%\}20cuenca.pdf.

[20] Anaya O. Caracterización morfométrica de la cuenca hidrográfica Chichao, Distrito de Chinchao, provincia Huanuco, región Huanuco. 2012. Available from: https://www.unas.edu.pe/web/sites/default/ files/web/archivos/actividades_academicas/CARACTERIZACION\%20MORFOMETRICA\%20DE\% 20LA\%20CUENCA\%2OHIDROGRAFICA\%20CHINCHAO\%2C\%2ODISTRITO\%20DE\%20CHINCHAO\% 2C\%2OPROVINCIA\%2ODE\%2OHUANUCO\%2C.pdf

[21] Jardí M. Forma de una cuenca de drenaje. Análisis de las variables morfométricas que nos la definen. 1985. Available from: https://www.raco.cat/index.php/RevistaGeografia/article/viewFile/45789/56812.

[22] Flores G, Lopez Z, Flores M. Método de análisis morfométrico de la red de drenaje de cuencas. 2016. Available from: https://es.slideshare.net/zulylopezhuachhua/analisis-morfometrico-de-una-cuenca-2.

[23] Strahler A. Quantitative analysis of watershed geomorphology. Civil Engineering. 1957.

[24] Londoño C. Cuencas hidrográficas: bases conceptuales-caracterizacionplanificacionadministración. 2001. Available from: https://www.academia.edu/29394172/CUENCAS_ HIDROGR\{\%\}C3\{\%\}81FICAS_BASES_CONCEPTUALES_CARACTERIZACI\{\%\}C3\{\%]93NPLANIFICACI\{\%\}C3\{\%\}93N-ADMINISTRACI\{\%\}C3\{\%\}93N.

[25] Romero A, López F. Morfometría de redes fluviales: revisión critica de los parámetros mas utilizados y aplicación al alto Guadalquivir. 1987.

[26] Sellers C, Buján S, Corbelle E, Miranda D. Morfología interpretativa de alta resolución usando datos LiDAR en la cuenca hidrográfica del río Paute en Ecuador. 2017. Available from: http://julianrojo.weebly. com/uploads/1/2/0/0/12008328/morfometria.pdf. 
[27] Meza M, Rodríguez A, Corvacho O, Tapia A. Análisis morfométrico de microcuencas afectadas por flujos de detritos bajo precipitación intensa en la quebrada de Camiña, norte grande de Chile. 2014. Available from: https://scielo.conicyt.cl/pdf/rda/n44/art03.pdf.

[28] Verdugo M. Análisis morfométrico de las microcuencas a las que pertenece el bosque y vegetación protectora Aguarongo (BVPA), influencia en el comportamiento hidrológico. 2017. Available from: https: //dspace.ups.edu.ec/bitstream/123456789/14381/1/UPS-CT007051.pdf.

[29] Rivas D, Moreno M. Delimitación hidrográfica y caracterización morfométrica de la cuenca del rio Metica Auxiliar de investigación. 2019. Available from: https://repository.ucc.edu.co/bitstream/20.500.12494/ 11104/6/2019_Delimitaci\{\%\}C3\{\%\}B3n_hidrogr\{\%\}C3\{\%\}A1fica_Rio_metica.pdf.

[30] Juárez I, Crettaz M, Gianello D, Juárez R. Análisis de las características morfométricas de la cuenca hidrográfica del arroyo Santa Bárbara para el diagnóstico ambiental. 2017. Available from: https://www.researchgate.net/profile/Melina_Crettaz_Minaglia/publication/320336304_Aportes_ del_analisis_de_las_caracteristicas_morfometricas_de_la_cuenca_hidrografica_del_arroyo_Santa_ Barbara_para_el_diagnostico_ambiental/links/59deb38c458515376b29f454/Ap.

[31] IGM. Capas de información geográfica básica del IGM de libre acceso. 2017. Available from: http: //www.geoportaligm.gob.ec/portal/index.php/descargas/cartografia-de-libre-acceso/registro/. 